\title{
Detection of Lower Tropospheric Responses to Solar Energetic Particles at Midlatitudes
}

\author{
K. A. Nicoll and R. G. Harrison \\ Department of Meteorology, University of Reading, P.O. Box 243, Reading, Berkshire RG6 6BB, United Kingdom
}

(Received 1 October 2013; published 2 June 2014)

\begin{abstract}
Solar energetic particles (SEPs) occasionally contribute additional atmospheric ionization beyond that arising from the usual galactic cosmic ray background. During an SEP event associated with a solar flare on April 11, 2013, the vertical ionization rate profile obtained using a balloon-borne detector showed enhanced ionization with a $26 \%$ increase at $20 \mathrm{~km}$, over Reading, United Kingdom. Fluctuations in atmospheric electrical parameters were also detected at the surface, beneath the balloon's trajectory. As no coincident changes in geomagnetism occurred, the electrical fluctuations are very likely to be associated with increased ionization, as observed by the balloon measurements. The lack of response of surface neutron monitors during this event indicates that energetic particles that are not detected at the surface by neutron monitors can nevertheless enter and influence the atmosphere's weather-generating regions.
\end{abstract}

DOI: 10.1103/PhysRevLett.112.225001

PACS numbers: 92.60.Pw, 96.50.Vg

Introduction.-Solar energetic particles (SEPs) are typically emitted during coronal mass ejections and solar flares with particles mostly consisting of protons with energies $E$ from $\mathrm{MeV}$ to tens of $\mathrm{GeV}$ [1]. For these energies the Earth's magnetosphere modulates particle penetration into the atmosphere (through the geomagnetic cutoff rigidity - the minimum rigidity required for a particle to reach a particular point in the magnetosphere) thus allowing greater particle flux at high latitude from the reduced geomagnetic shielding at the magnetic poles. The altitude down to which SEPs reach in the atmosphere is also energy dependent with most protons of energy less than $500 \mathrm{MeV}$ absorbed above $15 \mathrm{~km}$. Particles with $E>500 \mathrm{MeV}$ interact with atomic nuclei generating a cascade of secondary particles including neutrons that can be detected at ground level by neutron monitors during so-called ground level events (GLEs). Above the surface, lower energy SEPs can be detected by spacecraft e.g. from instrumentation on board Geostationary Operational Environmental Satellite (GOES), Advanced Composition Explorer (ACE), and Polar-orbiting Operational Environmental Satellite (POES). In addition a long series of balloon ionization measurements since 1958 has been performed by the Lebedev Physical Institute (LPI) [2] representing the only regular measurements of the in situ flux of ionizing particles between the surface and spacecraft altitudes.

Since SEP events cause a rapid enhancement of extraterrestrial ionization, they are a potential hazard for spacecraft systems as well as spacecraft crews. Enhanced

Published by the American Physical Society under the terms of the Creative Commons Attribution 3.0 License. Further distribution of this work must maintain attribution to the author(s) and the published article's title, journal citation, and DOI. ionization from SEPs has also been shown to affect atmospheric processes, influencing chemical reactions in the upper stratosphere by causing the destruction of ozone in polar regions, leading to changes in upper-level dynamics through changes in heating rates. For example, ozone concentrations in the northern polar atmosphere between 35 and $60 \mathrm{~km}$ were depleted by tens of percent following SEP events in 2003 [3]. In the lower atmosphere, ionization has been suggested to influence clouds, either by ioninduced nucleation of cloud condensation nuclei [4] or through cloud droplet charging arising from current flow in the global atmospheric electric circuit (GEC) $[5,6]$.

This paper presents measurements from a new ionization detector flying on a free balloon, during an SEP event on April 11, 2013. During the same event, substantial fluctuations were detected in surface atmospheric electricity measurements at a midlatitude site, but no GLE was detected. The measurements presented here not only demonstrate increased ionization down into the troposphere, but also an influence of energetic particles on atmospheric quantities already known to be associated with clouds.

Solar proton event characteristics.-The SEP event originated from an M6/3b solar flare on April 11, 2013, with a peak in the $\mathrm{x}$-ray flux detected at 0716UT. Enhancement of the $>100-\mathrm{MeV}$ proton flux began at 0940UT and the 10-MeV flux at 1055UT (using the criteria defined for GOES SEP event alerts). Figure 1 shows (a) GOES proton flux measurements and (b) terrestrial magnetic field measurements. There was no discernible perturbation of the geomagnetic field by the flare, supported by a planetary $K p$ index value of less than two throughout April 11 and April 12, 2013. The $>2-\mathrm{MeV}$ electron flux at geosynchronous orbit was also at normal to moderate levels throughout the period of interest. 


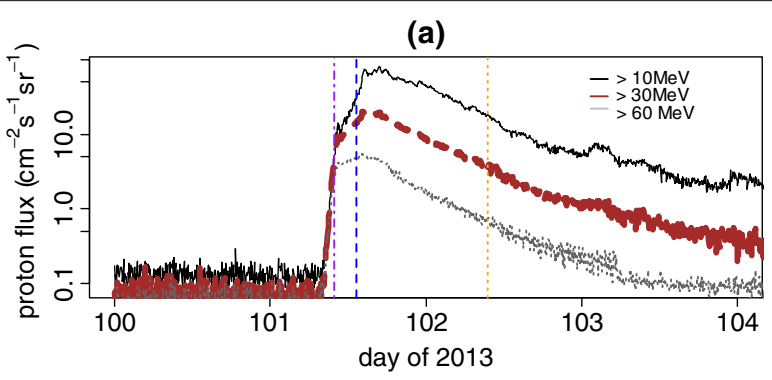

(b)

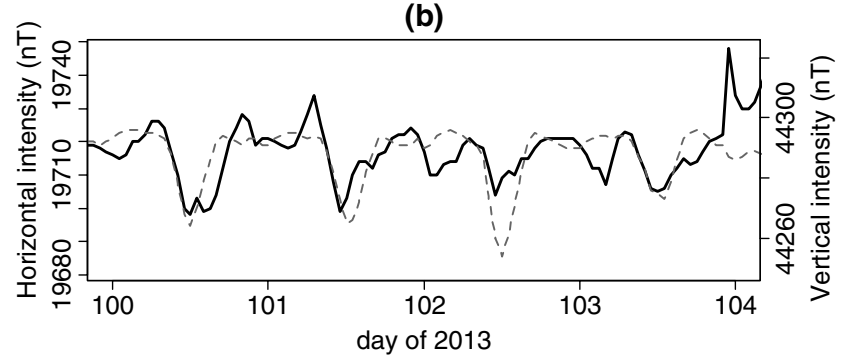

FIG. 1 (color online). (a) Time series of proton flux from GOES13 detectors around April 11, 2013 (year day 101), for proton energies $>10$ (black line), $>30$ (thick dashed brown line), and $>60 \mathrm{MeV}$ (gray line) (log scale). Vertical lines mark observed surface atmospheric electrical fluctuations on day 101 (dot-dashed line); Geigersonde launches on day 101 (flight 4) and 102 (flight 5) (dashed and dotted horizontal lines, respectively). (b) Time series of geomagnetic field at Hartland, United Kingdom, showing both horizontal (black line) and vertical components of magnetic field intensity (gray dashed line).

Ionization measurements.-Vertical profiles of ionization were obtained using two Geiger tube sensors [7] flown on a free balloon alongside a standard meteorological radiosonde. The "Geigersonde" device uses a compact high-tension supply for two miniature LND714 Geiger tubes, with an interval timing technique for improved resolution at low count rates. Data were returned through a Vaisala RS92 radiosonde using a PANDORA interface [8] together with pressure, temperature, relative humidity, and GPS position. Figure 2(a) shows typical vertical profiles of Geigersonde count rates of ionization during unperturbed (i.e., normal) atmospheric conditions, launched from Reading, United Kingdom $\left(51.45^{\circ} \mathrm{N}, 0.97^{\circ} \mathrm{W}\right)$. The ionization profile increases with height until reaching the Pfotzer maximum at $\sim 17 \mathrm{~km}$ (e.g., [2,9]]), with a maximum count rate during unperturbed conditions of $\sim 53$ counts per minute (cpm) (from the mean of all three unperturbed flights). Figure 2(b) shows measurements from two balloon flights performed during the SEP event, from 1319 to 1449UT (10-km altitude at 1355UT) on April 11 and from 0931 to 1242 UT (10-km altitude at 1030UT) on April 12, plotted alongside the mean of the measurements from the unperturbed days. It is evident that the Geiger count rates are increased during the SEP event, reaching a maximum of 67 cpm, with an enhancement from the maximum height reached $(25 \mathrm{~km})$ down to $\sim 10 \mathrm{~km}$, indicating penetration
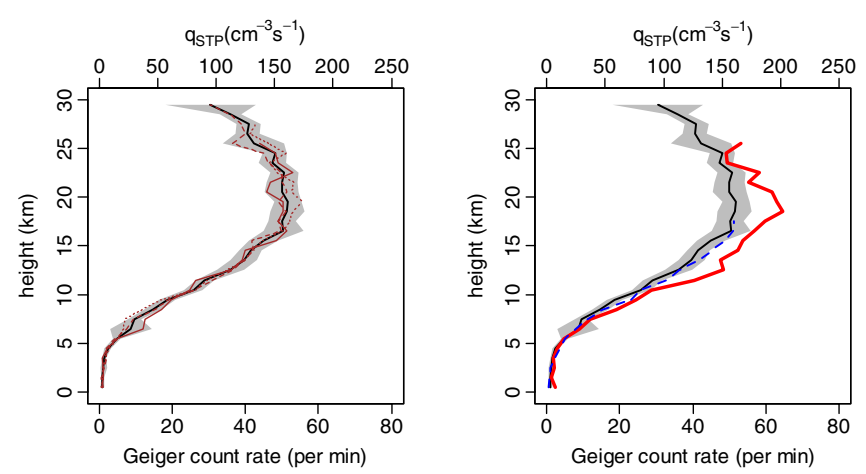

FIG. 2 (color online). Vertical profiles of Geiger count rates from Reading, United Kingdom (averaged on each flight for the two Geiger tubes carried and corrected for temperature), for soundings (a) reference flights during quiet atmospheric conditions and (b) after the solar flare of April 11, 2013 (red thick and dashed blue lines). In both plots, the black line shows the mean of the three flights in (a), with the gray shading representing the largest of \pm 1.96 standard errors from any of the three reference flights. A scale corresponding to the ion production rate at standard temperature and pressure, $q_{\mathrm{STP}}$, is also given.

of energetic particles well into the troposphere. Although the Geigersonde count rate is elevated on April 11, on April 12, it lies within the undisturbed range, despite the sustained enhanced proton flux measured at GOES [Fig. 1(a)]. Thus, the observations represent a transient response in atmospheric ionization and/or that the energies detected by the Geigersonde on April 11 were those of higher energy particles, which were no longer sufficiently enhanced to be detected against the typical variability.

Surface atmospheric electrical effects.-Figure 2(b) demonstrates that the SEP event on April 11 was associated with enhanced atmospheric ionization rates into the troposphere. One of several atmospheric properties influenced by changes in ionization is atmospheric electricity. Current flow in the atmosphere is described by the GEC [10], in which the conducting regions of Earth's surface and the ionosphere are regarded as two electrodes of a spherical capacitor, between which a leaky dielectric is formed from weakly ionized air. A potential difference $\left(V_{i}\right)$ of $\sim 300 \mathrm{kV}$ is generated between the ionosphere and Earth's surface by charge separation from thunderstorms and disturbed weather regions, permitting a conduction current, $J_{c}$, to flow vertically. $J_{c}$ depends on both $V_{i}$ and the electrical conductivity, $\sigma$, in a unit column of atmosphere from the surface to the ionosphere, with $\sigma$ varying with the ionization rate, $q$. Although a number of atmospheric electrical responses to short-term solar events have been reported in the literature (e.g., [11-13]), almost all reported measurements are during periods when geomagnetic variability accompanies enhanced ionization; hence, the results of magnetic or ionization influences are difficult to separate. The event described here consisted only of changes in proton flux, with no significant variations in geomagnetic 


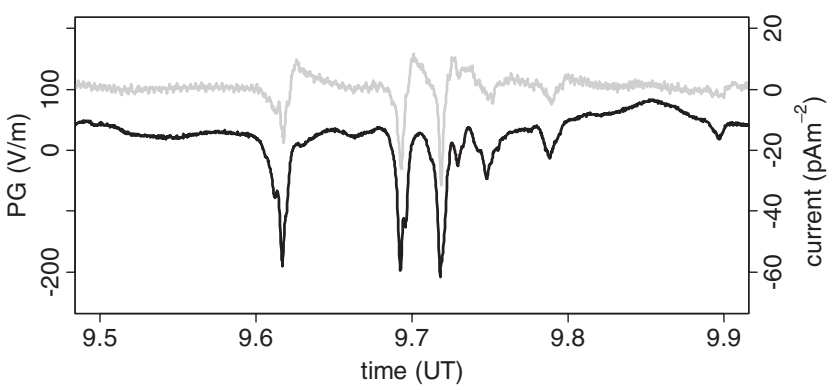

FIG. 3. Atmospheric electrical parameters measured from Reading, United Kingdom, on April 11, 2013, during an SEP event. PG is in black, and the vertical conduction current is in gray.

field. Thus, this event presents a rare opportunity to investigate the particle-related effect on atmospheric electrical parameters during solar disturbances.

Figure 3 shows measurements of surface atmospheric electrical parameters measured at Reading, United Kingdom, between 0900 and 1000UT on April 11, during the SEP event. The surface measurements were sampled at $1 \mathrm{~Hz}$. Potential gradient, PG, is measured using a Chubb JCI 131 electric field mill, set on a 0 to $2 \mathrm{kV} \mathrm{m}^{-1}$ range, pointing vertically upward. $\mathrm{PG}$ is defined as $+d V / d z$, where $V$ is the potential with respect to the Earth's surface, at a positive height $z$ above the surface. Vertical current flow is measured using the Geometrical Displacement and Conduction Current Sensor [14]. Figure 3 demonstrates that substantial changes occurred in the atmospheric electrical parameters at Reading during the SEP event. Both the PG and vertical conduction current show a reduction over a period of about 15 seconds, followed by a gradual recovery to fair-weather values (approximately $50 \mathrm{Vm}^{-1}$ for the PG and $1 \mathrm{pA} \mathrm{m}^{-2}$ for the conduction current).

To examine the cause of the electrical changes at Reading further, Figs. 4(b) and 4(c) show the 10-second standard deviation in (b) PG and point discharge, PD, and (c) vertical current. Point discharge, or corona, is measured using an upward-pointing vertical corona needle electrode, connected to a bipolar logarithmic current amplifier [15], and occurs when the local electric field around a vertically upward sharp point exceeds the threshold for local electrical breakdown in air (approximately $3 \mathrm{kV} \mathrm{m}^{-1}$ ). The fluctuations in all three electrical parameters occurred approximately 2 hours after the end of the flare period, coincident with the maximum rate of change of $>60 \mathrm{MeV}$ protons [Fig. 4(a)]. Since the maximum rate of change in the lower energy proton fluxes occurs later (0954UT and $1012 \mathrm{UT}$ for $>30-\mathrm{MeV}$ and $>10-\mathrm{MeV}$ protons, respectively), this suggests that the atmospheric electrical responses are primarily a result of ionization from the high-energy tail of the SEP particles. The large magnitude and transient nature of the electrical changes also suggest that electrostatic induction, rather than conduction, was responsible for the observed changes. This would be

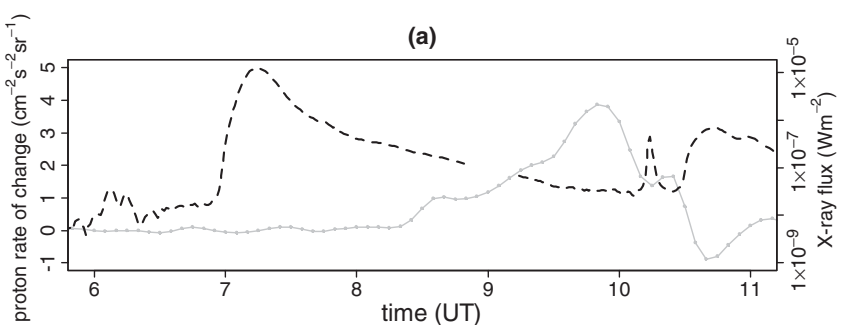

(b)

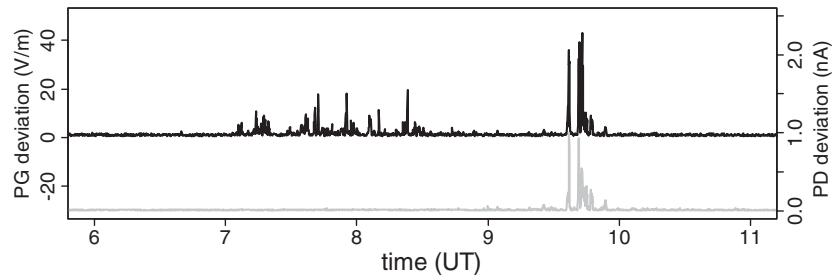

(c)

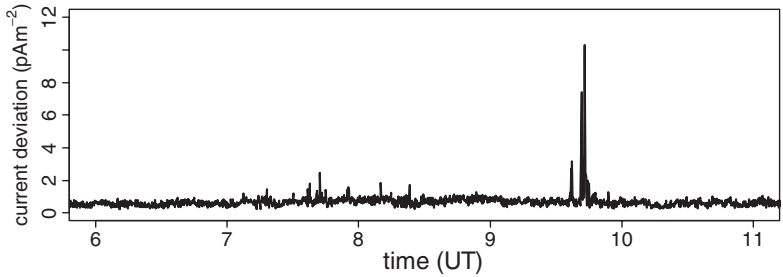

FIG. 4. (a) Rate of change of $>60 \mathrm{MeV}$ proton flux (gray solid line) and x-ray flux (black dashed line) from GOES 13 on April 11, 2013. (b) and (c) Ten-second standard deviations in surface atmospheric electrical measurements from Reading, United Kingdom. (b) PG, point discharge current (PD), and (c) vertical conduction current.

consistent with transient changes in the charge distribution above Reading occurring as a result of the increased ionization from high-energy SEP particles, inducing displacement currents in the suite of the atmospheric electrical instruments beneath.

Atmospheric electrical parameters can be sensitive to changes in local weather conditions, particularly concerning precipitation and wind; therefore, it is necessary to consider whether the fluctuations observed could have arisen solely from variability in the local weather. This is discussed further in the Supplemental Material [16], which demonstrates that no significant changes in meteorological conditions occurred during the disturbed period considered and also that the electrical variations could not be attributed to light rainfall. This analysis suggests that the fluctuations measured in atmospheric electrical parameters on April 11, 2013, were not due to local meteorological changes.

Discussion.-The results presented here demonstrate evidence of increased ionization in the atmosphere above Reading, United Kingdom, during an SEP event on April 11, 2013. Despite observed increases in the ionization rate at high altitudes, no increase was detected by the network of surface neutron monitors. This is surprising since given the relatively high geomagnetic cutoff rigidity of Reading (geomagnetic latitude $\sim 47.5^{\circ} \mathrm{N}$ ) the primary particles 
responsible for the increased ionization would require $E>2 \mathrm{GeV}$. Such particles would generate a cascade of secondary particles, including neutrons, detectable by surface neutron monitors; therefore, the absence of a response from surface neutron monitors suggests that the neutrons were not of sufficient number or energy to trigger the neutron monitors (likely to be related to the angular distribution of the incoming primary particles). Further, differences in the measurement technology between the neutron monitors [primarily sensitive to the hadronic (i.e., nucleonic) component of the secondary cascade] and Geigersonde tubes (mostly sensitive to the muon component) may account for some of the discrepancy between the two techniques. The typical number of GLEs recorded by neutron monitors during a solar cycle (around 12-15) is much less than the number of SEP events recorded from balloon measurements (22-23) [18]. Hence, the observations on April 11, 2013, may well represent another SEP event undetected by surface neutron monitors.

Despite the absence of a response in neutron monitors, an increase in the ionization rate during the SEP event was also detected by balloon measurements made by the LPI at polar latitudes. Measurements from Mirny, Antarctica at 0300UT on April 12 using a Geigersonde of different design showed increased ionization down to $\sim 27 \mathrm{~km}$ [19]. This supports the SEP event being capable of yielding increased atmospheric ionization, such as that observed over Reading during the earlier phase of the same event at 1319UT on April 11.

Substantial fluctuations were also observed in three independently observed surface atmospheric electrical parameters during the SEP event, which can be uniquely attributed to ionization effects, as no geomagnetic disturbances occurred during this period. We suggest that the changes in electrical parameters resulted from increased ionization from high-energy particles. The transient PG changes in Fig. 3 indicate episodic ionization generation and/or charge accumulation. The large magnitude of the electrical changes suggests an electrostatic origin, which may arise, for example, from a large amount of charge being suddenly delivered into the atmosphere over a substantial horizontal region. This would induce displacement currents in the atmospheric electrical sensors, which are proportional to the rate of change of PG, apparent if the PG and current time series (Fig. 3) are compared.

This work demonstrates that SEPs are not only capable of reaching altitudes in the lower atmosphere at midlatitudes but that atmospheric electrical parameters at the surface also undergo substantial associated changes. This solar-terrestrial coupling may have implications for a number of atmospheric processes, including charging of cloud droplets on the edges of layer clouds, which have been recently directly observed [20]. It follows that some SEP events provide opportunities in which to investigate the effect of ionization variability on lower atmosphere cloud processes.
Conclusions.-This paper presents, for the first time, simultaneous measurements of increased ionization in the troposphere, with a response in surface atmospheric electrical parameters at a midlatitude site during an SEP event. The absence of geomagnetic fluctuations allows the electrical variations to be attributed solely to ionization effects, for which coincident variability in high-energy particles $(>60 \mathrm{MeV})$ is observed. The variations in atmospheric electricity are consistent with an increase in lower troposphere conductivity, associated with enhanced ionization rates. Despite increased ionization detected outside Earth's atmosphere by GOES satellite instruments, no detection of increased ground level ionization was made by the worldwide neutron monitor network. This suggests that there are moderately energetic solar particles that have electrical effects within the weather-forming regions of the lower atmosphere but remain undetected in surface neutron monitors, presumably due to the low flux of energetic particles. Although the neutron monitor data provide a proxy for atmospheric ionization [21], these findings indicate that a gap exists in the current observational network of SEPs. Hence, the evaluation of the basic rate of influential SEP events may still neglect weak events causing atmospheric ionization. Our experimental approach illustrates that such space weather effects on the lower atmosphere could nevertheless be regularly and effectively observed using modified meteorological balloons.

The Geigersonde work was funded by the Science and Technology Facilities Council (airborne monitoring of space weather and radioactivity, ST/K001965/1). K. A. N. acknowledges support of the Leverhulme Trust through an Early Career Fellowship. R. Wilson and I. Read provided invaluable assistance with balloon launches and preparing prototypes, and Dr. A. Bennett and Professor G. Bazilevskya provided useful discussions. The BGS magnetic field data were obtained from http://www.geomag.bgs .ac.uk/data_service/data/obs_data/hourly_means.html and GOES 13 proton flux and x-ray flux from http:// www.swpc.noaa.gov/Data/index.html.

[1] G. A. Bazilevskaya, V. S. Makhmutov, Y. I. Stozhkov, A. K. Svirzheskaya, and N. S. Svirzhevsky, Adv. Space Res. 45, 603 (2010).

[2] Y. I. Stozhkov, N. S. Svirzhevsky, G. A. Bazilevskaya, A. N. Kvashnin, V. S. Makhmutov, and A. K. Svirzhevskaya, Adv. Space Res. 44, 1124 (2009).

[3] A. Seppälä, P. T. Verronen, E. Kyrölä, S. Hassinen, L. Backman, A. Hauchecorne, J. L. Bertaux, and D. Fussen, Geophys. Res. Lett. 31, L19107 (2004).

[4] H. Svensmark, J. Olaf P Pedersen, N. D. Marsh, M. B. Enghoff, and U. I. Uggerhøj, Proc. R. Soc. A 463, 385 (2007).

[5] B. A. Tinsley, Space Sci. Rev. 94, 231 (2000).

[6] M. J. Rycroft, K. A. Nicoll, K. L. Aplin, and R. G. Harrison, J. Atmos. Sol.-Terr. Phys. 90-91, 198 (2012). 
[7] R. G. Harrison, K. A. Nicoll, and A. G. Lomas, Rev. Sci. Instrum. 84, 076103 (2013).

[8] R. G. Harrison, K. A. Nicoll, and A. G. Lomas, Rev. Sci. Instrum. 83, 036106 (2012).

[9] R. G. Harrison, Rev. Sci. Instrum. 76, 126111 (2005).

[10] C. T. R. Wilson, Phil. Trans. R. Soc. A 221, 73 (1921).

[11] W. E. Cobb, Mon. Weather Rev. 95, 905 (1967).

[12] R. Holzworth and F. S. Mozer, J. Geophys. Res. 84, 2559 (1979).

[13] E. A. Kasatkina, O. I. Shumilov, M. J. Rycroft, F. Marcz, and A. V. Frank-Kamenetsky, Atmos. Chem. Phys. Discuss. 9, 21941 (2009).

[14] A. J. Bennett and R. G. Harrison, J. Atmos. Terr. Phys. 70, 1373 (2008).
[15] G. J. Marlton, R. G. Harrison, and K. A. Nicoll, Rev. Sci. Instrum. 84, 066103 (2013).

[16] See Supplemental Material at http://link.aps.org/ supplemental/10.1103/PhysRevLett.112.225001 for a summary of meteorological conditions at the time of the observed fluctuations in atmospheric electrical parameters, which includes Ref. [17].

[17] A. J. Bennett and R. G. Harrison, Phys. Rev. Lett. 111, 045003 (2013).

[18] G. A. Bazilevskaya, Adv. Space Res. 35, 458 (2005).

[19] G. A. Bazilevskaya (private communication).

[20] K. A. Nicoll and R. G. Harrison, Geophys. Res. Lett. 37, L13802 (2010).

[21] K. L. Aplin, R. G. Harrison, and A. J. Bennett, Adv. Space Res. 35, 1484 (2005). 